\title{
MELD scoring system: How golden is the gold.
}

\author{
Jayant Kumar ${ }^{1,2}$, Isabella Reccia ${ }^{1}$ \\ ${ }^{1}$ Department of Hepato-Pancreato-Biliary Surgery (HPB), Hammersmith Hospital, Imperial College, London, UK. \\ ${ }^{2}$ Faculty of Health and Science, Institute of Learning and Teaching, University of Liverpool, UK.
}

\section{Introduction}

The recognition of patients who can achieve best outcome following orthotropic liver transplantation (OLT) is quite challenging in transplantation science. Liver transplantation proffers the only curative option for patients with endstage liver disease (ESLD). Though, the supply of donor livers remains sparse to meet up the run on organs. Since its adaptation in 2002 the model for end-stage liver disease (MELD) score has become one of the most widely used systems for prioritizing liver transplant candidates throughout the world.

MELD is used to stratify candidates for liver transplantation based on objective measures of disease severity. It has been validated as a predictor of wait-list mortality in transplantation though it has been found to be predictor of outcome other surgeries chronic liver diseases [1]. Despite advancement in surgical techniques and perioperative care, the complications are quite high in emergency general surgery (EGS). These are reflected regarding increased rates of morbidity and mortality. These could be because of various disturbances in the physiological milieu of a body either due to present disease status such as peritonitis, blunt abdominal trauma, sepsis or on-going co-morbid malady. One of such condition is the chronic liver disease (CLD) which imposes a higher risk for developing post-operative complications independent of a type of surgery and disease process. The enormity of morbidity and mortality squares with the extent of hepatic decompensation [2].

Studies have shown that anaesthetic drugs have an adverse impact on the liver enzymes levels, which could be of little significance in a healthy individual. However, in patients with CLD these insults may precipitate hepatic decompensation [3]. In patients with CLD, Model for End-Stage Liver Disease (MELD) score has been evinced to correspond with the preoperative risk. This linear regression model has been designed on serum bilirubin and creatinine levels and international normalised ratio (INR). It is more objective and weights the variables; thereby even a slight increase in the MELD score makes an incremental input into the menace [4]. Even though this system was moulded to foresee mortality following TIPS, later it was also enacted to stratify patients ahead of liver transplantation, to predict perioperative mortality post-transplant. Lately, researchers have found a notable link allying MELD score and mortality in trauma surgery patients [5].

The published works of literature on post-operative outcomes in surgeries other than the transplant are based on retrospective cohorts. There has been consistent increase in operative risk with CLD undergoing any kind surgeries. However, there is a lack of any meta-analysis or systemic review. These cultivating evinces and inefficiency of existent models, e.g. the American College of Surgeons National Surgical Quality Improvement Project Surgical Risk Calculator, connote a complete openness for MELD score; that may be indispensable in envisioning outcomes in a number of patients not going through a liver transplant procedures [6]. There have been various other models of post-operative outcomes in the transplant patients were reported but none of them has shown clear cut reproducibility [7]. Considering these facts we are here discussing pit and falls of these scoring system and other possible options.

First, is that the inclusion of the Deyo-Charlson index, which has been widely used to assess the burden of chronic illness and predict outcomes? A study by Poses et al. have valued its importance in prognostication in ICU patients by adding them to a more physiological system such as Acute Physiology and Chronic Health Evaluation II (APACHE II) [8]. However, in a very landmark study by Quach et al. have reported the limited role of the Deyo-Charlson index in predicting mortality in ICU patients [9]. However, this index may have practical applications when another physiological scoring system has not been prospectively determined. Studies have outlined the importance of Charlson index in the assessment of outcomes beyond hospital discharge in non-ICU settings thus further studies are needed to explore the ability of this scoring system in ICU patients.

The second salient point to mention is the need for analysing predictability of MELD scoring system against other prognostic scores as APACHE II), Sequential organ failure assessment (SOFA), Child-Turcotte-Pugh (CTP). Referencing the study by Dusseja et al. over 100 patients with acute on chronic liver disease, which showed that APACHE II scoring system is superior to other prognostic scores in predicting its short-term mortality [10]. Here I like to quote a prospective study by Theocharidou et al. involving a cohort of 635 patients with cirrhosis admitted to ICU. Where they have 
developed a Royal Free Hospital (RFH), a score for disease prognostication and mortality prediction and compared it against other prognostic models like APACHE II, MELD, CTP and Chronic Liver Failure-Sequential Organ Failure Assessment (CLIF-SOFA) model. Although they have reported good discriminative ability and calibration like other though this also needs further external validation [11].

The third point in the panel of this editorial is making allowance for the role serum lactate and standard base deficit in the praxis scoring system of CLD patients admitted to the intensive care unit is useful for risk assessment, prognostication and foretelling in-hospital mortality [12]. By the same token, need and duration of mechanical ventilation, hypoalbuminemia, anaemia, blood transfusion, the length of hospital stay are few other factors which are laid in various studies as a contributor of poorer long-term survival [13]. The quintessential requirements that ideal prognostication model should fulfil are; it must be established on easily measurable parameters, non-invasive, clinically sound, and its validity should be generalizable to a variegated congregation of population. Albeit, many groups have put forwarded various systems with promising internal outcomes, however, external validation with reference to aetiology, gender, ethnicity, geography is lacking in the real world.

Since the appearance of the MELD modus operandi in 2002, MELD has been legitimated and enacted to a vast majority of clinical situations encountered by CLD patients. The enforcement of MELD scoring to prioritise donor livers for transplant in 2002 had called forth a diminution in waiting list registrations and scaled down the mortality on the waiting list without grieving post-transplant outcomes. The MELD score helps clinicians to risk stratifying various interventions on a daily basis in patients with CLD in addition to influencing treatment options [14]. The MELD scoring system does have its foibles and require further reinforcement by other measures of liver, or global functioning is imperative to boost may its prognostic accuracy in the CLD patient undergoing emergency general surgery.

\section{References}

1. Malinchoc M, Kamath PS, Gordon FD, et al. A model to predict poor survival in patients undergoing transjugular intrahepatic portosystemic shunts. Hepatology 2000; 31: 864-871.

2. Havens JM, Peetz AB, Do WS, et al. The excess morbidity and mortality of emergency general surgery. J Trauma Acute Care Surg. 2015; 78: 306-311.
3. Gholson CF, Provenza JM, Bacon BR. Hepatologic considerations in patients with parenchymal liver disease undergoing surgery. Am J Gastroenterol 1990; 85: 487-496.

4. O'Leary JG, Friedman LS. Predicting surgical risk in patients with cirrhosis: From art to science. Gastroenterology. 2007; 132: 1609-1611.

5. Peetz A, Salim A, Askari R, et al. Association of model for end-stage liver disease score and mortality in trauma patients with chronic liver disease. JAMA Surg 2016; 151: 41-48.

6. Bilimoria KY, Liu Y, Paruch JL, et al. Development and evaluation of the universal ACS NSQIP surgical risk calculator: A decision aid and informed consent tool for patients and surgeons. J Am Coll Surg 2013; 217: 23-34.

7. Cholongitas E, Marelli L, Shusang V, et al. A systematic review of the performance of the model for end-stage liver disease (MELD) in the setting of liver transplantation. Liver Transpl 2006; 12: 1049-1061.

8. Poses RM, McClish DK, Smith WR, et al. Prediction of survival of critically ill patients by admission comorbidity. J Clin Epidemiol 1996; 49: 743-747.

9. Quach S, Hennessy D, Faris P, et al. A comparison between the APACHE II and Charlson Index Score for predicting hospital mortality in critically ill patients. BMC Health Serv Res 2009; 9: 129.

10. Duseja A, Choudhary NS, Gupta S, et al. APACHE II score is superior to SOFA, CTP and MELD in predicting the shortterm mortality in patients with acute-on-chronic liver failure (ACLF). J Dig Dis 2013; 14: 484-490.

11. Theocharidou E, Pieri G, Mohammad AO, et al. The royal free hospital score: A calibrated prognostic model for patients with cirrhosis admitted to intensive care unit. Comparison with current models and CLIF-SOFA score. Am J Gastroenterol 2014; 109: 554-562.

12. Tobias AZ, Guyette FX, Seymour CW, et al. Pre-resuscitation Lactate and Hospital Mortality in Prehospital Patients. Prehospital Emerg Care 2014; 18: 321-327.

13. Han ML, Chen CC, Kuo SH, Hsu WF, Liou JM, Wu MS, et al. Predictors of in-hospital mortality after acute variceal bleeding in patients with hepatocellular carcinoma and concurrent main portal vein thrombosis. J Gastroenterol Hepatol 2014; 29: 344-351.

14. Kamath PS, Kim WR. The model for end-stage liver disease (MELD). Hepatology 2007; 45: 797-805.

\section{Correspondence to:}

Kumar Jayant,

$\mathrm{MD}, \mathrm{MS}$,

Department of Hepato-Pancreato-Biliary Surgery (HPB),

Hammersmith Hospital,

Imperial College,

London,

UK.

E-mail: drjayantsun19@gmail.com 